\title{
The g-based Jordan Algebra and Lie Algebra with Application to the Model of Visco-Elastoplasticity
}

Chein-Shan Liu

Associate Professor, Department of Mechanical and Marine Engineering, National Taiwan Ocean University, Keelung, Taiwan, R.O.C.

Follow this and additional works at: https://jmstt.ntou.edu.tw/journal

Part of the Mechanical Engineering Commons

\section{Recommended Citation}

Liu, Chein-Shan (2001) "The g-based Jordan Algebra and Lie Algebra with Application to the Model of Visco-

Elastoplasticity," Journal of Marine Science and Technology. Vol. 9: Iss. 1, Article 1.

DOI: 10.51400/2709-6998.2429

Available at: https://jmstt.ntou.edu.tw/journal/vol9/iss1/1

This Research Article is brought to you for free and open access by Journal of Marine Science and Technology. It has been accepted for inclusion in Journal of Marine Science and Technology by an authorized editor of Journal of Marine Science and Technology. 


\title{
THE $g$-BASED JORDAN ALGEBRA AND LIE ALGEBRA WITH APPLICATION TO THE MODEL OF VISCO-ELASTOPLASTICITY
}

\author{
Chein-Shan Liu
}

Key words: Visco-elastoplasticity, Large deformation, Lorentz group, Consistency scheme.

\begin{abstract}
By a matrix representation the $g$-based Jordan algebra [1] is proved to form a $g$-based Lie algebra under the commutator product. Then we derive a new dynamical system based on the composition of the $g$-based Jordan and Lie algebras, which possesses internal symmetry group $\operatorname{DSO}_{o}(n, 1)$, and its projection $\operatorname{PDSO}_{o}(n, 1)$. Utilizing this concept we obtain a linear representation of a constitutive model of visco-elastoplasticity with large deformation. The irreducible representation in the vector space admits of the projective dilation proper orthochronous Lorentz group $P D S O_{o}(5,1)$ in the visco-elastoplastic phase and the dilation special Euclidean group $D S E(5)$ in the viscoelastic phase. The input path and the relaxation time decide that when the symmetry switches between the two groups. Based on such symmetry a numerical scheme which satisfies the consistency condition for every time step is devised, which preserves the internal symmetry $\operatorname{PDSO}_{o}(5,1)$ of the model in the visco-elastoplastic phase so as to locate the stress point automatically on the yield surface at each time step without iterations at all.
\end{abstract}

\section{INTRODUCTION}

In the last few decades there has been a great interest in formulating the nonlinear mechanical system with noncanonical or generalized bracket formulations. To each mechanics there associates at least one algebraic structure determined by the proper bracket of the theory. The new algebra still preserves the Lie algebra axioms. Along this way a trilinear Jordan algebra has been formed to formulate a particular dynamical system with nonvanishing associator in [1]. The algebraic foundation of this dynamical system is the non-associative algebra $[2,3]$. Some applications include the perfect elastoplasticity [4,5], the magnetic spin equation [6], the suspension particle orientation equation [7], and so on. In this paper we first proceed to examine such type dynamical systems from the view point of Lie

Paper Received Dec. 11, 2000. Author for Correspondence: Chein-Shan Liu. *Associate Professor, Department of Mechanical and Marine Engineering, National Taiwan Ocean University, Keelung, Taiwan, R.O.C. algebra and Lie group. Then we derive a new dynamical system based on the composition of the $g$-based Jordan and Lie algebras, which will prove to has internal symmetry group $\operatorname{DSO}_{o}(n, 1)$ in the homogeneous coordinates space.

We will soon find that the aforementioned group theory is useful for the foundation of the constitutive model of visco-elastoplasticity with large deformation. For such model the underlying algebra is thus revealed. An internal symmetry of a constitutive model is a statement that when one makes changes in the states of the model, a particular expression for certain constitutive phenomena he formulates does not change. The changes or transformations he makes to the constitutive model which leave the form of the expression unchanged are naturally linked with the invariance of a conserved quantity. A numerical scheme which preserves symmetry and utilizes the invariance property from one time stop to the next one or few stops will be more capable of capturing key features during plastic deformation and has long-term stability and much improved efficiency and accuracy. Therefore the issue of internal symmetries in constitutive laws of plasticity is not only important in its own right, but will also find great applications to computational plasticity.

Hong and Liu recently found symmetry groups in a constitutive model of perfect elastoplasticity [4], in a constitutive model of bilinear elastoplasticity [8], as well as in a constitutive model of large deformation perfect elastoplasticity with the Jaumann stress rate [9]. The latter work is then extended by Liu and Hong [10] to the constitutive models of large deformation perfect elastoplasticity with corotational stress rates, where we have compared the effects of different corotational stress rates on the model behavior, giving a sufficient criterion for the non-oscillation response under the simple shear deformation. It is found that using internal symmetry inherent in the constitutive model a consistency scheme can be developed. One direct benefit of such scheme is that the stress point is automatically updated on the yield surface without iterative calculations for every time step. This is what the conventional constitutive 
numerical schemes, which were directly formulated in the stress space, desired and failed to achieve. As we know that the numerical schemes developed up to now for the integration of the constitutive equations of plasticity are executed in the stress space. In order to enforce the consistency condition at every time step the conventional algorithms require some iterative calculations to force the stress point at the end of each time step to converge to the yield surface, which is known as a main source of numerical errors and of consumption of computational time. Here basing on the $g$-based dynamical system theory we will extend the results in [9] to the constitutive model of visco-elastoplasticity with large deformation.

\section{A $g$-BASED JORDAN ALGEBRA}

Let $\mathbf{X}^{s} \cdot \mathbf{Y}^{s}$ an inner product of $\mathbf{X}^{s}$ and $\mathbf{Y}^{s}$ on a vector space $\mathbb{V}$ over $\mathbb{R}$. Let $\mathbb{M}:=\mathbb{R} \oplus \mathbb{V}$ the vector space direct sum of $\mathbb{R}$ and $\mathbb{V}$, and for each $X \in \mathbb{M}$ we assign

$$
x:=X^{0}+g \mathbf{X}^{s},
$$

where $X^{0} \in \mathbb{R}$ is a scalar part, $\mathbf{X}^{s} \in \mathbb{V}$ is a vector part, and $g$ with $g^{2}:=g \cdot g=1$ has been named the $g$-number in [1].

For $x=X^{0}+g \mathbf{X}^{s}, \quad y=Y^{0}+g \mathbf{Y}^{s} \in \mathbb{M}$, we define the addition of $X$ and $y$ by

$$
x+y:=\left(X^{0}+Y^{0}\right)+g\left(\mathbf{X}^{s}+\mathbf{Y}^{s}\right),
$$

and the product by

$$
\begin{aligned}
X \mathcal{Y} & =\left(X^{0}+g \mathbf{X}^{s}\right)\left(Y^{0}+g \mathbf{Y}^{s}\right):=\left(X^{0} Y^{0}+\mathbf{X}^{s} \cdot \mathbf{Y}^{s}\right) \\
& +g\left(X^{0} \mathbf{Y}^{s}+Y^{0} \mathbf{X}^{s}\right) .
\end{aligned}
$$

For all $x, \mathcal{Y}, z \in \mathbb{M}$ and all $a \in \mathbb{R}$, the algebra introduced has the following properties:

$$
\begin{aligned}
& {[x, y]=x y-y x=\mathbf{0}} \\
& x(y+z)=x y+x z \\
& (x+y) z=x z+y z \\
& a(x+y)=a x+a y, \\
& a(x y)=(a x) y=x(a y) \\
& {[x, y, z]:=(x y) z-x(y z)=g\left[\mathbf{X}^{s}, \mathbf{Y}^{s}, \mathbf{Z}^{s}\right] \neq \mathbf{0},} \\
& {\left[x^{2}, y, x\right]=\mathbf{0}}
\end{aligned}
$$

where

$$
\begin{aligned}
& {\left[\mathbf{X}^{s}, \mathbf{Y}^{s}, \mathbf{Z}^{s}\right]:=\mathbf{X}^{s} \cdot \mathbf{Y}^{s} \mathbf{Z}^{s}-\mathbf{Z}^{s} \cdot \mathbf{Y}^{s} \mathbf{X}^{s}} \\
& \forall \mathbf{X}^{s}, \mathbf{Y}^{s}, \mathbf{Z}^{s} \in \mathbb{V}
\end{aligned}
$$

is the associator defined in $\mathbb{V}$ (see, e.g. [1]). Eq. (4) is the commutativity of product, Eq. (5) the left-distributivity of product, Eq. (6) the right-distributivity of product, Eq. (7) the distributivity of scalar multiple, Eq. (8) the associativity of scalar multiple, Eq. (9) the nonassociativity of triple product, and Eq. (10) the Jordan identity. An algebraic system with commutativity (4) and the Jordan identity (10) is known as a (commutative) Jordan algebra $[2,3]$. Usually, we choose $\mathbb{V}:=\mathbb{R}^{n}, n \geq$ 2 , to denote the $n$-dimensional Euclidean space. For the special case with $n=1$ the non-associativity in Eq. (9) disappears and thus the resulting algebra is associative, of which the numbers $a+g b$, with $a, b \in \mathbb{R}$, have been named the double numbers by Yaglom [11].

\section{A $g$-BASED LIE ALGEBRA}

Let us identify

$$
x \rightarrow \mathbf{X}:=\left[\begin{array}{c}
\mathbf{X}^{s} \\
X^{0}
\end{array}\right],
$$

and consider the following map:

$$
\mathrm{M}: X \rightarrow \mathrm{M}(X):=\left[\begin{array}{ll}
X^{0} \mathbf{I}_{n} & \mathbf{X}^{s} \\
\left(\mathbf{X}^{s}\right)^{t} & X^{0}
\end{array}\right] .
$$

For each $X$ we not only assign a column matrix representation $\mathbf{X}$ with order $(n+1) \times 1$ but also a matrix representation $\mathrm{M}(X)$ with order $(n+1) \times(n+1)$. Thus, the product operation in Eq. (3) can be equivalently written as

$$
\mathbf{M}(X) \mathbf{Y}=\left[\begin{array}{cc}
X^{0} \mathbf{I}_{n} & \mathbf{X}^{s} \\
\left(\mathbf{X}^{s}\right)^{t} & X^{0}
\end{array}\right]\left[\begin{array}{l}
\mathbf{Y}^{s} \\
Y^{0}
\end{array}\right]=\left[\begin{array}{c}
X^{0} \mathbf{Y}^{s}+Y^{0} \mathbf{X}^{s} \\
X^{0} Y^{0}+\mathbf{X}^{s} \cdot \mathbf{Y}^{s}
\end{array}\right]
$$

under the correspondences: $x \leftrightarrow \mathrm{M}(X)$ and $\mathcal{Y} \leftrightarrow \mathbf{Y}$. The symbol $\leftrightarrow$ signifies the two-way relation.

Let us investigate the matrix commutator of $\mathrm{M}(X)$ and $\mathrm{M}(\mathscr{Y})$ by

$$
\begin{array}{r}
{[\mathrm{M}(x), \mathrm{M}(\mathscr{y}):=\mathrm{M}(x) \mathrm{M}(\mathscr{y})-\mathrm{M}(\mathscr{y}) \mathrm{M}(x)=} \\
{\left[\begin{array}{cr}
\mathbf{X}^{s}\left(\mathbf{Y}^{s}\right)^{t}-\mathbf{Y}^{s}\left(\mathbf{X}^{s}\right)^{t} & \mathbf{0} \\
\mathbf{0} & 0
\end{array}\right] .}
\end{array}
$$

Obviously, we have 


$$
\mathrm{M}(X) \mathrm{M}(\mathscr{y}) \neq \mathrm{M}(\mathscr{y}) \mathrm{M}(x),
$$

which is different from the commutative property (4) of the Jordan algebra. Furthermore, using Eq. (15), we can prove that for all $x \in \mathbb{M}$ the corresponding $\mathrm{M}(X) \in$ $\mathbb{R}^{(n+1) \times(n+1)}$ form a Lie algebra, because of

$[\mathrm{M}(X), \mathrm{M}(\mathscr{y})]$, bilinear in $\mathrm{M}(X)$ and $\mathrm{M}(\mathscr{y})$

$[\mathrm{M}(X), \mathrm{M}(\mathscr{Y})]=-[\mathrm{M}(\mathscr{Y}), \mathrm{M}(X)]$, skew-symmetric,

$[[\mathrm{M}(x), \mathrm{M}(y)], \mathrm{M}(z)]+[[\mathrm{M}(z), \mathrm{M}(x)], \mathrm{M}(y)]$

$+[[\mathrm{M}(y), \mathrm{M}(z)], \mathrm{M}(x)]=\mathbf{0}$, the Jacobi identity.

Applying the commutator in Eq. (15) to $\mathbf{Z}$, we obtain

$$
[\mathrm{M}(x), \mathrm{M}(\mathscr{y})] \mathbf{Z}=\left[\begin{array}{c}
\mathbf{Y}^{s} \cdot \mathbf{Z}^{s} \mathbf{X}^{s}-\mathbf{X}^{s} \cdot \mathbf{Z}^{s} \mathbf{Y}^{s} \\
0
\end{array}\right],
$$

from which and Eq. (9), we thus identify the following two operations:

$$
[\mathrm{M}(X), \mathrm{M}(\mathscr{Y})] \mathrm{Z} \leftrightarrow[\mathcal{Y}, Z, X]
$$

Here, the non-associativity of the Jordan algebra implies the non-commutativity of the Lie algebra, and vice versa.

\section{A $g$-BASED DYNAMICAL SYSTEM} $[1]$,

An associator equation system has been defined in

$$
\dot{x}=[\mathcal{Y}, Z, \mathcal{Z}] .
$$

From Eqs. (12) and (20), the above system can be re-expressed by

$$
\dot{\mathbf{X}}=[\mathrm{M}(\mathcal{U}), \mathrm{M}(\mathscr{Y})] \mathbf{Z} .
$$

Note that $[\mathrm{M}(\mathcal{U}), \mathrm{M}(\mathscr{Y})]$ is skew-symmetric, and also that $X^{0}$ is a constant, say $X^{0}=1$, because the last element on the right-hand side by Eq. (19) is zero.

We consider another dynamical system and give its applications in the next section:

$$
\dot{x}=s x .
$$

Let $X=X^{0}+g \mathbf{X}^{s}$ and $\mathcal{S}=S^{0}+g \mathbf{S}^{s}$ and substitute them into the above equation, yielding

$$
\dot{X}^{0}+g \dot{\mathbf{X}}^{s}=S^{0} X^{0}+\mathbf{S}^{s} \cdot \mathbf{X}^{s}+g\left(S^{0} \mathbf{X}^{s}+X^{0} \mathbf{S}^{s}\right) .
$$

Equating the scalar and vector parts, respectively, we get

$$
\begin{aligned}
& \dot{X}^{0}=S^{0} X^{0}+\mathbf{S}^{s} \cdot \mathbf{X}^{s}, \\
& \dot{\mathbf{X}}^{s}=S^{0} \mathbf{X}^{s}+X^{0} \mathbf{S}^{s} .
\end{aligned}
$$

Let

$$
\mathbf{Q}:=\frac{\mathbf{X}^{s}}{X^{0}}
$$

be the nonhomogeneous coordinates. Using Eqs. (25) and (26), we obtain the governing equation for $\mathbf{Q}$,

$$
\dot{\mathbf{Q}}=\mathbf{S}^{s}-\left(\mathbf{S}^{s} \cdot \mathbf{Q}\right) \mathbf{Q} .
$$

Using the identification (12) and the map $\mathrm{M}(\mathcal{S})$ in Eq. (13), Eq. (23) can also be written as

$$
\dot{\mathbf{X}}=\mathrm{M}(\mathcal{S}) \mathbf{X},
$$

where the control tensor $\mathrm{M}(\mathcal{S})$ is read as

$$
\mathrm{M}(S)=\left[\begin{array}{ll}
\mathcal{S}^{0} \mathbf{I}_{n} & \mathbf{S}^{s} \\
\left(\mathbf{S}^{s}\right)^{t} & S^{0}
\end{array}\right] .
$$

It is symmetric and is a Lie algebra as shown in Eqs. (16)-(18). Equation (29) can be combined to the associator equation (22) but specialized to

$$
\dot{\mathbf{X}}=[\mathrm{M}(y), \mathrm{M}(z)] \mathbf{X},
$$

where

$$
[\mathrm{M}(\mathscr{Y}), \mathrm{M}(Z)]=\left[\begin{array}{cc}
\mathbf{Y}^{s}\left(\mathbf{Z}^{s}\right)^{t}-\mathbf{Z}^{s}\left(\mathbf{Y}^{s}\right)^{t} & \mathbf{0} \\
\mathbf{0} & 0
\end{array}\right]
$$

obtained from Eq. (15) is skew-symmetric. Thus, we may introduce a differential equation system based on the combination of the Jordan and Lie algebras:

$$
\dot{\mathbf{X}}=\mathbf{A X},
$$

where

$$
\begin{gathered}
\mathbf{A}:=\mathrm{M}(\mathcal{S})+[\mathrm{M}(\mathcal{Y}), \mathrm{M}(Z)]= \\
{\left[\begin{array}{cc}
S^{0} \mathbf{I}_{n}+\mathbf{Y}^{s}\left(\mathbf{Z}^{s}\right)^{t}-\mathbf{Z}^{s}\left(\mathbf{Y}^{s}\right)^{t} & \mathbf{S}^{s} \\
\left(\mathbf{S}^{s}\right)^{t} & S^{0}
\end{array}\right] .}
\end{gathered}
$$

In terms of the nonhomogeneous coordinates $\mathbf{Q}$ 
defined in Eq. (27), from Eqs. (33) and (34) we obtain

$$
\dot{\mathbf{Q}}=\left[\mathbf{Y}^{s}\left(\mathbf{Z}^{s}\right)^{t}-\mathbf{Z}^{s}\left(\mathbf{Y}^{s}\right)^{t}\right] \mathbf{Q}+\mathbf{S}^{s}-\left(\mathbf{S}^{s} \cdot \mathbf{Q}\right) \mathbf{Q} .
$$

It is an extension of Eq. (28) to include the spin term [ $\mathbf{Y}^{s}$ $\left.\left(\mathbf{Z}^{s}\right)-\mathbf{Z}^{s}\left(\mathbf{Y}^{s}\right)^{t}\right] \mathbf{Q}$ on the right-hand side. The spin matrix $\mathbf{Y}^{s}\left(\mathbf{Z}^{s}\right)^{t}-\mathbf{Z}^{s}\left(\mathbf{Y}^{s}\right)^{t}$ has $2 n$ parameters, but the general spin matrix with order $n \times n$ has $n(n-1) / 2$ independent parameters. Let $n(n-1) / 2=2 n$, we obtain $n=5$. It is easy to see that the above $\mathbf{A}$ satisfies

$$
\mathbf{A}^{t} \mathbf{g}+\mathbf{g A}=2 S^{0} \mathbf{g}
$$

where

$$
\mathbf{g}:=\left[\begin{array}{ll}
\mathbf{g}_{s s} & \mathbf{g}_{s 0} \\
\mathbf{g}_{0 s} & \mathbf{g}_{00}
\end{array}\right]=\left[\begin{array}{cc}
\mathbf{I}_{n} & \mathbf{0}_{n \times 1} \\
\mathbf{0}_{1 \times n} & -1
\end{array}\right]
$$

is the Minkowski metric (in the space-like convention). The $(n+1)$-dimensional vector space of homogeneous coordinates $\mathbf{X}$ endowed with the Minkowski metric $\mathbf{g}$ is referred to as Minkowski spacetime $\mathbb{M}^{n+1}$. Thus, the single parameter subgroup generated by $\mathbf{A}$ has the following transformation action:

$$
\mathbf{X}(t)=\exp \left[\int_{0}^{t} S^{0}(\eta) d \eta\right] \mathbf{G}(t) \mathbf{X}(0),
$$

where $\int_{0}^{t} S^{0}(\eta) d \eta$ controls the dilation/contraction of $\mathbf{X}$, and $\mathbf{G}$ is an element of the proper orthochronous Lorentz group $\mathrm{SO}_{o}(n, 1)$ satisfying

$$
\mathbf{G}^{t} \mathbf{g G}=\mathbf{g},
$$

$$
\operatorname{det} \mathbf{G}=1 \text {, }
$$

$$
G^{0}{ }_{0}>0
$$

Really, Zeeman [12] was able to show that the causality assumption in the Minkowski spacetime $\mathbb{M}^{n+1}$ renders a composition of a translation, a dilation, and a proper orthochronous Lorentz transformation. Here we concentrate on the last two effects on material modeling and denote the composition of dilation and proper orthochronous Lorentz transformation by $\mathrm{DSO}_{o}$ $(n, 1)$, whose projection denoted by $\operatorname{PDSO}_{o}(n, 1)$ is thus an internal symmetry group of the dynamical system (35). It deserves to note that the $\mathbf{A}$ in Eq. (33) may be dependent on $t$ as well as on $\mathbf{X}$. No matter what case it is, we call such $\mathbf{A}$ the Lie algebra of $d s o(n, 1)$ if Eq. (36) is fulfilled.

\section{THE CONSTITUTIVE MODEL OF VISCO-ELASTOPLASTICITY}

The constitutive law of elastoplasticity of solid materials proposed by Prandtl [13] and Reuss [14] can be re-formulated and enlarged to take account of large deformation ( $c f$. Hong and Liu [9]) and viscosity effect:

$$
\begin{aligned}
& \mathbf{D}=\mathbf{D}^{e}+\mathbf{D}^{p}, \\
& \stackrel{\circ}{\mathbf{s}}=2 G \mathbf{D}^{e}-\frac{1}{\eta} \mathbf{s}, \\
& \mathbf{s} \dot{\gamma}^{p}=2 \tau_{y} \mathbf{D}^{p}, \\
& \|\mathbf{s}\| \leq \sqrt{2} \tau_{y}, \\
& \dot{\gamma}^{p} \geq 0, \\
& \|\mathbf{s}\| \dot{\gamma}^{p}=\sqrt{2} \tau_{y} \dot{\gamma}^{p},
\end{aligned}
$$

in which the three material constants, namely the shear modulus $G$, the relaxation time $\eta$ and the shear yield stress $\tau_{y}$, are determined experimentally and all are assumed to be positive. The second equation in the above is really a generalization of the Maxwell type viscoelasticity constitutive equation [15]. The relaxation time characterizes one of the viscoelastic properties of the material. The bold-faced symbols $\mathbf{D}, \mathbf{D}^{e}, \mathbf{D}^{p}$ and $\mathbf{s}$ stand for the deviatoric parts of the deformation rate, elastic deformation rate, plastic deformation rate, and Cauchy's stress, respectively, all being symmetric and traceless tensors, whereas $\gamma^{p}$ is a scalar, called the equivalent shear plastic strain. All the $\mathbf{D}, \mathbf{D}^{e}, \mathbf{D}^{p}, \mathbf{s}$ and $\gamma^{p}$ are functions of time $t$.

A superimposed dot denotes (material) differentiation with respect to time, that is $d / d t$, and a surmounted circle "o" on $\mathbf{s}$ represents a Lie derivative of $\mathbf{s}$ with respect to $\mathbf{W}$, that is the Jaumann rate

$$
\stackrel{\circ}{\mathbf{S}}:=\dot{\mathbf{s}}-\mathbf{W s}+\mathbf{s W} .
$$

Here $\mathbf{W}$ is the spin tensor, defined as the skew-symmetric part of the velocity gradient. A dot is placed between two tensors to denote their Euclidean inner product, and as usual the Euclidean norm of a tensor, s say, is represented by $\|\mathbf{s}\|:=\sqrt{\mathbf{S} \cdot \mathbf{s}}$.

\section{A Nonlinear Representation}

We first analyze the constitutive model (42)-(47). Substituting Eqs. (43), (44) and (48) into Eq. (42), we obtain

$$
\dot{\mathbf{s}}-\mathbf{W} \mathbf{s}+\mathbf{s} \mathbf{W}+\frac{1}{\eta} \mathbf{s}+\frac{1}{\gamma_{y}} \dot{\gamma}^{p} \mathbf{s}=2 G \mathbf{D},
$$


where

$$
\gamma_{y}:=\frac{\tau_{y}}{G}
$$

is the shear yield strain.

Define

$$
X^{0}:=\exp \left(\frac{\gamma^{p}}{\gamma_{y}}\right),
$$

and then Eq. (49) becomes

$$
\frac{d}{d t}\left(X^{0} \mathbf{s}\right)-X^{0} \mathbf{W} \mathbf{s}+X^{0} \mathbf{s} \mathbf{W}+\frac{1}{\eta} X^{0} \mathbf{s}=2 G X^{0} \mathbf{D} .
$$

The inner product of $\mathbf{s}$ with Eq. (49) is

$$
\mathbf{s} \cdot \dot{\mathbf{s}}+\left[\frac{\dot{\gamma}^{p}}{\gamma_{y}}+\frac{1}{\eta}\right] \mathbf{s} \cdot \mathbf{s}=2 G \mathbf{s} \cdot \mathbf{D} .
$$

Hence

$$
\|\mathbf{s}\|=\sqrt{2} \tau_{y} \Rightarrow \tau_{y} \dot{\gamma}^{p}+\frac{\gamma_{y} \tau_{y}}{\eta}=\mathbf{s} \cdot \mathbf{D} .
$$

Recalling $\tau_{y}>0$ and $\eta>0$, we have

$$
\|\mathbf{s}\|=\sqrt{2} \tau_{y} \Rightarrow\left\{\mathbf{s} \cdot \mathbf{D}>\frac{\gamma_{y} \tau_{y}}{\eta} \Leftrightarrow \dot{\gamma}^{p}>0\right\}
$$

which asserts that

$$
\left\{\|\mathbf{s}\|=\sqrt{2} \tau_{y} \text { and } \mathbf{s} \cdot \mathbf{D}>\frac{\gamma_{y} \tau_{y}}{\eta}\right\} \Rightarrow \dot{\gamma}^{p}>0 .
$$

Conversely, if $\dot{\gamma}^{p}>0$, Eq. (47) assures $\|\mathbf{s}\|=\sqrt{2} \tau_{y}$, which together with Eq. (55) asserts that

$$
\dot{\gamma}^{p}>0 \Rightarrow\left\{\|\mathbf{s}\|=\sqrt{2} \tau_{y} \text { and } \mathbf{s} \cdot \mathbf{D}>\frac{\gamma_{y} \tau_{y}}{\eta}\right\} \text {. }
$$

Statements (56) and (57) tell us that the yield condition $\|\mathbf{s}\|=\sqrt{2} \tau_{y}$ and the straining condition $\mathbf{s} \cdot \mathbf{D}>$ $\gamma_{y} \tau_{y} / \eta$ are sufficient and necessary for plasticity $\dot{\gamma}^{p}>0$. In view of Eqs. (45), (46) and (54), the two statements are logically equivalent to the following criteria:

$$
\dot{\gamma}^{p}= \begin{cases}\frac{1}{\tau_{y}} \mathbf{s} \cdot \mathbf{D}-\frac{\gamma_{y}}{\eta}>0 & \text { if }\|\mathbf{s}\|=\sqrt{2} \tau_{y} \text { and } \mathbf{s} \cdot \mathbf{D}>\frac{\gamma_{y} \tau_{y}}{\eta}, \\ 0 & \text { if }\|\mathbf{s}\|<\sqrt{2} \tau_{y} \text { or } \mathbf{s} \cdot \mathbf{D} \leq \frac{\gamma_{y} \tau_{y}}{\eta} .\end{cases}
$$

From Eqs. (49), (58) and (50) follows a two-phase nonlinear system of differential equations:

$$
\dot{\mathbf{s}}-\mathbf{W} \mathbf{s}+\mathbf{s} \mathbf{W}=\left\{\begin{array}{rr}
\left(\frac{1}{\eta}-\frac{\mathbf{s} \cdot \mathbf{D}}{\tau_{y} \gamma_{y}}\right) \mathbf{s}-\frac{1}{\eta} \mathbf{s}+2 G \mathbf{D} \text { if }\|\mathbf{s}\|=\sqrt{2} \tau_{y} \\
-\frac{1}{\eta} \mathbf{s}+2 G \mathbf{D} & \text { and } \mathbf{s} \cdot \mathbf{D}>\frac{\gamma_{y} \tau_{y}}{\eta}, \\
\text { if }\|\mathbf{s}\|<\sqrt{2} \tau_{y}
\end{array}\right.
$$

According to criteria (58) and the complementary trios (45)-(47) and further to the two-phase system (59), the model of visco-elastoplasticity has precisely two phases: the on (or visco-elastoplastic) phase in which $\dot{\gamma}^{p}>0$ and $\|\mathbf{s}\|=\sqrt{2} \tau_{y}$ and the off (or viscoelastic) phase in which $\dot{\gamma}^{p}=0$ and $\|\mathbf{s}\| \leq \sqrt{2} \tau_{y}$. In the viscoelastoplastic phase the plasticity mechanism is on so that the model exhibits visco-elastoplastic behavior, while in the off phase the plasticity mechanism is off so that the model responds viscoelastically. Thus, Eq. (58) is called the on-off switching criteria for the mechanism of plasticity, and Eq. (59) is a nonlinear differential representation of the constitutive model.

\section{Stress Bound in the Viscoelastic Phase}

Here we say something about the bound of the stress in the viscoelastic phase. At the first glance, we may be confused by the two conditions $\|\mathbf{s}\|<\sqrt{2} \tau_{y}$ or $\mathbf{s} \cdot$ $\mathbf{D} \leq \gamma_{y} \tau_{y} / \eta$ listed in Eq. (59) $)_{2}$ for the viscoelasticity. May we question that is there any loading process with sufficiently small $\mathbf{D}$ such that $\mathbf{s} \cdot \mathbf{D} \leq \gamma_{y} \tau_{y} / \eta$ holds for any given $\eta$ and admissible initial stress to render the stress over the bound after a long time elapsed, and thus contradicts to the inequality (45)? In order to tackle this problem, let us take the inner product of $\mathbf{s}$ with Eq. $(59)_{2}$, yielding

$$
\frac{1}{2} \frac{d}{d t}\|\mathbf{s}\|^{2}+\frac{1}{\eta}\|\mathbf{s}\|^{2}=2 G \mathbf{s} \cdot \mathbf{D} .
$$

Under the condition $\mathbf{S} \cdot \mathbf{D} \leq \gamma_{y} \tau_{y} / \eta$ we obtain

$$
\frac{d}{d t}\|\mathbf{s}\|^{2}+\frac{2}{\eta}\|\mathbf{s}\|^{2} \leq \frac{4 \tau_{y}^{2}}{\eta} .
$$

Integrating the avove inequality from $t_{i}$ to $t$ gives

$$
\|\mathbf{s}(t)\|^{2} \leq 2 \tau_{y}^{2}+\left(\left\|\mathbf{s}\left(t_{i}\right)\right\|^{2}-2 \tau_{y}^{2}\right) \exp \left[\frac{-2}{\eta}\left(t-t_{i}\right)\right] .
$$

We thus conclude that for any admissible initial stress $\left\|\mathbf{s}\left(t_{i}\right)\right\| \leq \sqrt{2} \tau_{y}$ at an initial time $t_{i}$, it should be

$$
\|\mathbf{s}(t)\|^{2} \leq \sqrt{2} \tau_{y}^{2}, \quad \forall t \geq t_{i}
$$

Therefore, we not need to worry that the stress will exceed the yield bound in the viscoelastic phase. For the considered model herein, the rate effect is included to take account of. If the value of $\|\mathbf{D}\|$ is too small such that $\|\mathbf{D}\| \leq \gamma_{y} /(\sqrt{2} \eta)$ and thus $\mathbf{s} \cdot \mathbf{D} \leq \gamma_{y} \tau_{y} / \eta$ for all admissible stress, then even the initial stress is chosen to be located on the yield surface, the subsequent stress responses are always in the fashion of viscoelasticity. However, for more large $\mathbf{D}$ the visco-elastoplastic phase may be initiated. In the last subsection we will give a calculating example of this sort. 


\section{A Linear Representation}

Due to the vanishing traces of the deviatoric tensors $\mathbf{S}$ and $\mathbf{D}$, i.e.,

$$
s^{33}=-s^{11}-s^{22}, D^{33}=-D^{11}-D^{22},
$$

there only have five independent equations in Eq. (52). To delete this redundancy, let

$$
\mathbf{X}=\left[\begin{array}{c}
\mathbf{X}^{s} \\
X^{0}
\end{array}\right]=\left[\begin{array}{c}
X^{1} \\
X^{2} \\
X^{3} \\
X^{4} \\
X^{5} \\
X^{0}
\end{array}\right]:=\frac{X^{0}}{\tau_{y}}\left[\begin{array}{c}
a_{1} s^{11}+a_{2} s^{22} \\
a_{3} s^{11}+a_{4} s^{22} \\
s^{23} \\
s^{13} \\
s^{12} \\
\tau_{y}
\end{array}\right]
$$

where

$a_{1}:=\sin \left(\theta+\frac{\pi}{3}\right), a_{2}:=\sin \theta, a_{3}:=\cos \left(\theta+\frac{\pi}{3}\right), a_{4}:=\cos \theta$,

with $\theta$ being any real number, and let

$$
\mathbf{A}_{s}^{0}=\left(\mathbf{A}_{0}^{s}\right)^{t}
$$

where

$$
\mathbf{A}_{0}^{s}=\left[\begin{array}{c}
A_{0}^{1} \\
A_{0}^{2} \\
A_{0}^{3} \\
A_{0}^{4} \\
A_{0}^{5}
\end{array}\right]:=\frac{2}{\gamma_{y}}\left[\begin{array}{c}
a_{1} D_{11}+a_{2} D_{22} \\
a_{3} D_{11}+a_{4} D_{22} \\
D_{23} \\
D_{13} \\
D_{12}
\end{array}\right] .
$$

Thus, it is obvious that

$$
\mathbf{A}^{0} \mathbf{X}^{s}=\frac{X^{0}}{\gamma_{y} \tau_{y}} \mathbf{s} \cdot \mathbf{D}
$$

Furthermore, it follows from Eqs. (51), (58) 1 and (69) that

$$
\frac{\dot{X}^{0}}{X^{0}}=\frac{1}{\gamma_{y} \tau_{y}} \mathbf{s} \cdot \mathbf{D}-\frac{1}{\eta}=\frac{1}{X^{0}} \mathbf{A}_{s}^{0} \mathbf{X}^{s}-\frac{1}{\eta} \cdot
$$

Therefore, the on-off switching criteria (58) turn out to be

$$
\dot{X}^{0}= \begin{cases}\mathbf{A}_{s}^{0} \mathbf{X}^{s}-\frac{X^{0}}{\eta}>0 & \text { if } \mathbf{X}^{t} \mathbf{g X}=0 \text { and } \mathbf{A}_{0}^{s} \mathbf{X}^{s}>\frac{X^{0}}{\eta}, \\ 0 & \text { if } \mathbf{X}^{t} \mathbf{g X}<0 \text { or } \mathbf{A}_{s}^{0} \mathbf{X}^{s} \leq \frac{X^{0}}{\eta},\end{cases}
$$

where

$$
\mathbf{g}=\left[\begin{array}{ll}
\mathbf{g}_{s s} & \mathbf{g}_{s 0} \\
\mathbf{g}_{0 s} & \mathbf{g}_{00}
\end{array}\right]=\left[\begin{array}{cc}
\mathbf{I}_{5} & \mathbf{0}_{5 \times 1} \\
\mathbf{0}_{1 \times 5} & -1
\end{array}\right]
$$

The condition $\mathbf{X}^{t} \mathbf{g X}=0$ is used to replace the yield condition $\|\mathbf{s}\|^{2}=2 \tau_{y}^{2}$, because of $X^{0}>0$ and

$$
\mathbf{X}^{t} \mathbf{g X}=\left(X^{0}\right)^{2}\left(\frac{\|s\|^{2}}{2 \tau_{y}^{2}}-1\right)
$$

by Eqs. (65) and (72). Moreover, the inequality (45) is now transformed to the inequality $\mathbf{X}^{t} \mathbf{g X} \leq 0$.

Consequently, Eqs. (52) and (71) are combined together as follows:

$$
\dot{\mathbf{X}}=\mathbf{A X}
$$

where

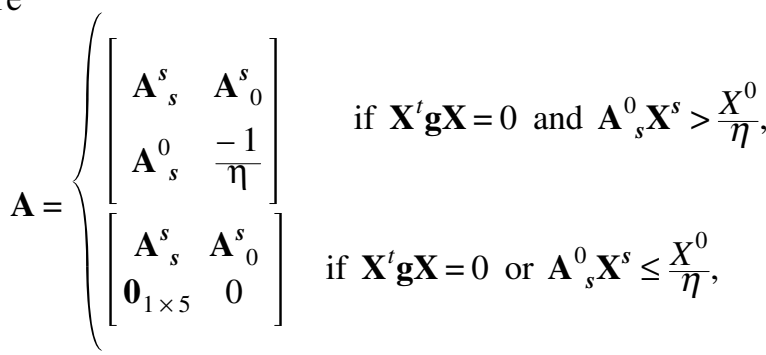

in which

$$
\mathbf{A}_{s}^{s}:=\left[\begin{array}{ccccc}
\frac{-1}{\eta} & 0 & 2 a_{2} W_{23} 2 a_{1} W_{13} & 2\left(a_{1}-a_{2}\right) W_{12} \\
0 & \frac{-1}{\eta} & 2 a_{4} W_{23} 2 a_{3} W_{13} & 2\left(a_{3}-a_{4}\right) W_{12} \\
-2 a_{2} W_{23} & -2 a_{4} W_{23} & \frac{-1}{\eta} & -W_{12} & -W_{13} \\
-2 a_{1} W_{13} & -2 a_{3} W_{13} & W_{12} & \frac{-1}{\eta} & -W_{23} \\
2\left(a_{2}-a_{1}\right) W_{12} & 2\left(a_{4}-a_{3}\right) W_{12} & W_{13} & W_{23} & \frac{-1}{\eta}
\end{array}\right]
$$

After deleting the diagonal terms, $\mathbf{A}_{s}^{s}$ is skew-symmetric.

From Eqs. (72) and (74)-(76) it follows that

$$
\begin{aligned}
\frac{1}{2} \frac{d}{d t}\left[\left(\mathbf{X}^{s}\right)^{t} \mathbf{g}_{s s} \mathbf{X}^{s}\right] & =\left(\mathbf{X}^{s}\right)^{t}\left[\mathbf{A}_{s}^{s} \mathbf{X}^{s}+\mathbf{A}_{0}^{s} X^{0}\right] \\
& =\frac{-1}{\eta}\left(\mathbf{X}^{s}\right)^{t} \mathbf{X}^{s}+X^{0} \mathbf{A}_{s}^{0} \mathbf{X}^{s} .
\end{aligned}
$$

Thus, the on-off switching criteria (71) can be further refined to

$$
\dot{X}^{0}= \begin{cases}\mathbf{A}_{s}^{0} \mathbf{X}^{s}-\frac{X^{0}}{\eta}>0 & \text { if } \mathbf{X}^{t} \mathbf{g X}=0 \text { and } \frac{d}{d t}\left[\left(\mathbf{X}^{s}\right)^{t} \mathbf{g}_{s s} \mathbf{X}^{s}\right]>0, \\ 0 & \text { if } \mathbf{X}^{t} \mathbf{g X}<0 \text { or } \frac{d}{d t}\left[\left(\mathbf{X}^{s}\right)^{t} \mathbf{g}_{s s} \mathbf{X}^{s}\right] \leq 0 .\end{cases}
$$

Hence in the augmented stress space what corresponds to the yield condition $\|\mathbf{s}\|=\sqrt{2} \tau_{y}$ is the cone condition $\mathbf{X}^{t} \mathbf{g X}=0$ and what corresponds to the straining condi- 
tion $\mathbf{s} \cdot \mathbf{D}>\gamma_{y} \tau_{y} / \eta$ is the growing "spatial" coordinate condition $d\left[\left(\mathbf{X}^{s}\right)^{t} \mathbf{g}_{s s} \mathbf{X}^{s}\right] / d t>0$.

Note that Eq. (74) is a linear, irreducible, $(5+1)$ dimensional representation of the constitutive model (42)-(47), in which $\mathbf{X}$ and $\mathbf{A}$ are the augmented stress vector and the control tensor, respectively. The control tensor A organizes the input information of the deviatoric deformation rate tensor $\mathbf{D}$ (normalized with respect to the shear yield strain $\gamma_{y} / 2$ ), the spin tensor $\mathbf{W}$, as well as the relaxation time parameter $\eta$. If Eq. (74) is viewed as a matrix representation, the $(5+1) \times 1$ matrix $\mathbf{X}$ contains the contravariant components of the augmented stress vector $\mathbf{X}$, and the $(5+1) \times(5+1)$ matrix $\mathbf{A}$ contains the mixed components of the control tensor $\mathbf{A}$.

\section{The Cone and the Discs}

Here we study the properties of the augmented stress space of $\mathbf{X}$ induced by the constitutive model (42)-(47). The model formulated in the deviatoric stress space of $\mathbf{s}$ may be converted into a model in the augmented stress space of $\mathbf{X}$; the first five equations of Eq. (74) and Eqs. (47), (45) and (46) thus become successively

$$
\left[\begin{array}{cc}
\mathbf{I}_{5} & \mathbf{0}_{5 \times 1} \\
\mathbf{0}_{1 \times 5} & \mathbf{X}^{t} \mathbf{g X}
\end{array}\right] \dot{\mathbf{X}}=\left[\begin{array}{cc}
\mathbf{A}_{s}^{s} & \mathbf{A}^{s} \\
\mathbf{0}_{5 \times 1} & 0
\end{array}\right] \mathbf{X}
$$

$$
\mathbf{X}^{t} \mathbf{g X} \leq 0
$$

$$
\dot{X}^{0} \geq 0
$$

The vector space of augmented stresses $\mathbf{X}$ endowed with the Minkowski metric tensor $\mathbf{g}$ in Eq. (72) is referred to as Minkowski spacetime and designated as $\mathbb{M}^{5+1}$.

Thus, a deviatoric stress point $\mathbf{s}$ on the yield hypersphere $\|\mathbf{s}\|=\sqrt{2} \tau_{y}$ in Euclidean space $\mathbb{E}^{5}$ according to Eq. (73) corresponds to an augmented stress point $\mathbf{X}$ on the right circular cone $\left\{\mathbf{X} \mid \mathbf{X}^{t} \mathbf{g X}=0\right\}$ emanating from $\mathbf{X}=0$ of Minkowski spacetime $\mathbb{M}^{5+1}$, henceforth referred to as the cone, while an $\mathbf{s}$ within the yield hypersphere corresponds to an $\mathbf{X}$ in the interior $\{\mathbf{X} \mid$ $\left.\mathbf{X}^{t} \mathbf{g X}<0\right\}$ of the cone. The exterior $\left\{\mathbf{X} \mid \mathbf{X}^{t} \mathbf{g X}>0\right\}$ of the cone is uninhabitable since $\|\mathbf{s}\|=\sqrt{2} \tau_{y}$ is forbidden according to Eq. (45). When $X^{0}$ is frozen in the viscoelastic phase as indicated by Eq. $(77)_{2}$, the augmented stress $\mathbf{X}$ stays in the closed 5-disc $\mathbb{D}^{5}$ (i.e. closed 5-ball $\left.\mathbb{B}^{5}\right)$ on the hyperplane $X^{0}=$ constant in the space of $\left(X^{1}\right.$, $\left.X^{2}, X^{3}, X^{4}, X^{5}, X^{0}\right)$; the hyperplane is identified to be Euclidean 5 -space $\mathbb{E}^{5}$, which is endowed with the Euclidean metric $\mathbf{I}_{5}$. In summary, the augmented stress $\mathbf{X}$ either evolves on the cone when in the visco-elastoplastic phase, or stays in the discs of simultaneity, which are stacked up one by one in the interior of the cone and are glued to the cone, when in the viscoelastic phase. This inspires us to remould the spacetime by removing the interior of the cone and gluing (identifying) a continuously infinite number of stacking Euclidean closed 5discs to the cone. This surgery results in a composite space endowed with the Minkowski metric (72) on the cone and the Euclidean metric $\mathbf{I}_{5}$ on the closed discs.

\section{5. $\operatorname{PDSO}_{o}(5,1)$ Symmetry in the Visco-elastoplastic Phase}

The solution of Eq. (74) can be expressed in the following formula for the transition from the augmented stress $\mathbf{X}\left(t_{1}\right)$ at time $t_{1}$ to the augmented stress $\mathbf{X}(t)$ at time $t$ :

$$
\mathbf{X}(t)=\exp \left[\left(t_{1}-t\right) / \eta\right]\left[\mathbf{G}(t) \mathbf{G}^{-1}\left(t_{1}\right)\right] \mathbf{X}\left(t_{1}\right)
$$

in which $\mathbf{G}(t)$ is a transformation tensor satisfying

$$
\begin{aligned}
& \dot{\mathbf{G}}(t)=\mathbf{A}^{\prime}(t) \mathbf{G}(t), \\
& \mathbf{G}(0)=\mathbf{I}_{6},
\end{aligned}
$$

where

$$
\mathbf{A}^{\prime}=\mathbf{A}+\frac{1}{\eta} \mathbf{I}_{6}
$$

is the deviatoric part of $\mathbf{A}$.

In what follows we concentrate on the viscoelastoplastic phase to bring out internal symmetry inherent in the model. Denote by $\mathrm{I}_{\text {on }}$ an open, maximal, continuous time interval during which the mechanism of plasticity is on exclusively. From Eqs. (75) 1 , (76) and (72) it is easy to verify that the control tensor $\mathbf{A}$ in the visco-elastoplastic phase is a special case of Eq. (34) with $n=5$ and $S^{0}=-1 / \eta$, satisfying

$$
\mathbf{A}^{t} \mathbf{g}+\mathbf{g A}=\frac{-2}{\eta} \mathbf{g}
$$

Hence, the corresponding transformation is a subgroup of $\operatorname{DSO}_{o}(5,1)$.

From Eq. $(77)_{1}, \dot{X}^{0}>0$ strictly when the mechanism of plasticity is on; hence,

$$
\mathrm{X}^{0}(t)>\mathrm{X}^{0}\left(t_{1}\right), \quad \forall t>t_{1}, \quad t, t_{1} \in \mathrm{I}_{\mathrm{on}},
$$

which means that there exists future-pointing timeorientation from the augmented stress $\mathbf{X}\left(t_{1}\right)$ to $\mathbf{X}(t)$. Moreover, such time-orientation is a causal one, because the augmented stress transition formula (81) and inequality (86) establish a causality relation between the two augmented stresses $\mathbf{X}\left(t_{1}\right)$ and $\mathbf{X}(t)$ in the sense that the preceding augmented stress $\mathbf{X}\left(t_{1}\right)$ influences the 
following augmented stress $\mathbf{X}(t)$ according to formula (81). Accordingly, the augmented stress $\mathbf{X}\left(t_{1}\right)$ chronologically and causally precedes the augmented stress $\mathbf{X}(t)$.

We solve Eq. (39) for the inverse

$$
\mathbf{G}^{-1}=\mathbf{g G} t \mathbf{g}
$$

and partition $\mathbf{G}$ as

$$
\mathbf{G}=\left[\begin{array}{ll}
\mathbf{G}_{s}^{s} & \mathbf{G}_{0}^{s} \\
\mathbf{G}_{s}^{0} & G_{0}^{0}
\end{array}\right],
$$

where $\mathbf{G}_{s}^{s}, \mathbf{G}_{0}^{s}$ and $\mathbf{G}_{s}^{0}$ are of order $5 \times 5,5 \times 1$ and $1 \times$ 5 , respectively. Thus, Eq. (81) is partitioned into the following matrix equation:

$$
\begin{aligned}
& {\left[\begin{array}{l}
\mathbf{X}^{s}(t) \\
X^{0}(t)
\end{array}\right]=\exp \left[\left(t_{1}-t\right) / \eta\right]}
\end{aligned}
$$

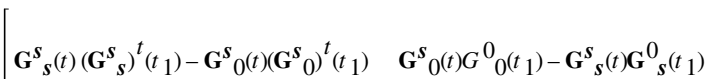

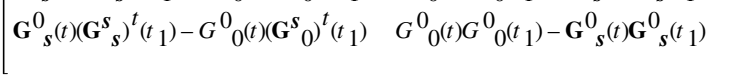

$$
\begin{aligned}
& {\left[\begin{array}{l}
\mathbf{X}^{s}\left(t_{1}\right) \\
X^{0}\left(t_{1}\right)
\end{array}\right]}
\end{aligned}
$$

which is valid for the visco-elastoplastic phase.

How can one determine the deviatoric stress tensor $\mathbf{s}(t)$ once he has the augmented stress vector $\mathbf{X}(t)$ ? From Eq. (65) it follows that

$$
\left[\begin{array}{l}
s^{11} \\
s^{22} \\
s^{23} \\
s^{13} \\
s^{12}
\end{array}\right]=\left[\begin{array}{ccc}
a_{4} & -a_{2} & \mathbf{0}_{2 \times 3} \\
-a_{3} & a_{1} & \sqrt{3} \\
\mathbf{0}_{3 \times 2} & \frac{\sqrt{3}}{2} \mathbf{I}_{3}
\end{array}\right] \frac{2 \tau_{y}}{\sqrt{3} X^{0}} \mathbf{X}^{s} .
$$

This is indeed a projective realization of the response. By this and the visco-elastoplastic phase transition formula (89) one can map $\mathbf{s}\left(t_{1}\right)$ to the current response $\mathbf{s}(t)$.

\section{DSE (5) Symmetry in the Viscoelastic Phase}

Contrary to Eq. (89) of the visco-elastoplastic phase, the transition formula for the viscoelastic phase is very simple. To find it, recall that Eqs. (81)-(83) are still applicable but with

$$
\mathbf{G}(t)=\left[\begin{array}{cc}
\mathbf{R}(t) & \mathbf{T}(t) \\
\mathbf{0}_{1 \times 5} & 1
\end{array}\right],
$$

where $\mathbf{R}$ and $\mathbf{T}$ are respectively of order $5 \times 5$ and $5 \times 1$ and governed by

$$
\begin{aligned}
& \dot{\mathbf{R}}=\mathbf{A}^{s}{ }_{s} \mathbf{R}, \\
& \dot{\mathbf{T}}=\mathbf{A}^{s}{ }_{s} \mathbf{T}+\mathbf{A}^{\prime s},
\end{aligned}
$$

where

$$
\mathbf{A}^{s}{ }_{s}=\mathbf{A}_{s}^{s}+\frac{1}{\eta} \mathbf{I}_{5}
$$

is the deviatoric part of $\mathbf{A}_{s}^{s}$, which is given in Eq. (76), and $\mathbf{A}_{0}^{s_{0}}$ is given in Eq. (68). Thus it is easy to show that $\mathbf{R} \in S O(5), \mathbf{T} \in T(5)$, and $\mathbf{G} \in S E(5)$; therefore, the constitutive law in the viscoelastic phase has an internal symmetry characterized by the group $D S E(5)$, which is the semi-direct product of the translation group $T(5)$ with the dilation and the proper rotation group $D S O(5)$.

The inverse of Eq. (91) is given by

$$
\mathbf{G}^{-1}=\left[\begin{array}{cc}
\mathbf{R}^{t} & -\mathbf{R}^{t} \mathbf{T} \\
\mathbf{0}_{1 \times 5} & 1
\end{array}\right] .
$$

Thus according to Eq. (81) we obtain

$$
\begin{aligned}
& {\left[\begin{array}{l}
\mathbf{X}^{s}(t) \\
X^{0}(t)
\end{array}\right]=} \\
& \left.\begin{array}{c}
\exp \left[\left(t_{1}-t\right) / \eta\right] \mathbf{R}(t) \mathbf{R}^{t}\left(t_{1}\right) \\
\mathbf{0}_{1 \times 5}-\exp \left[\left(t_{1}-t\right) / \eta\right] \mathbf{R}(t) \mathbf{R}^{t}\left(t_{1}\right) \mathbf{T}\left(t_{1}\right)+\mathbf{T}(t) \\
1
\end{array}\right] \\
& {\left[\begin{array}{l}
\mathbf{X}^{s}\left(t_{1}\right) \\
X^{0}\left(t_{1}\right)
\end{array}\right]}
\end{aligned}
$$

which is valid for the viscoelastic phase. In a similar way the stress response in the viscoelastic phase can be realized by invoking Eq. (90) and the viscoelastic phase transition formula (94).

In summary the stress response is the projective realization (90) of the visco-elastoplastic phase transition formula (89) or the viscoelastic phase transition formula (94); switching between the two depends upon the control tensor $\mathbf{A}$ and obeys the on-off switching criteria (71). The switching from a $D S O_{o}(5,1)$ group in the visco-elastoplastic phase to a $\operatorname{DSE}(5)$ group in the viscoelastic phase indicates that internal symmetry switches from one kind to another, and vice versa. As a result the constitutive model in the deviatoric stress space of $\mathbf{s}$ has symmetry switching between group $D S E$ (5) acting on the closed 5-ball of admissible states and the projective dilation and the proper orthochronous Lorentz group $P D S O_{o}(5,1)$ acting on the yield hypersphere.

\section{A Consistency Scheme}

For numerical calculation we first discretize Eq. (81) as follows: 


$$
\mathbf{X}_{n+1}=\exp [-\Delta t / \eta]\left[\mathbf{G}\left(t_{n+1}\right) \mathbf{G}^{-1}\left(t_{n}\right)\right] \mathbf{X}_{n},
$$

where $\mathbf{X}_{n}$ denotes the value of $\mathbf{X}$ at the discrete time stop $t_{n}$ and $\Delta t$ is the time increment, that is, $\Delta t=t_{n+1}-t_{n}$. The simplest scheme for the approximation of $\mathbf{G}\left(t_{n+1}\right) \mathbf{G}^{-1}$ $\left(t_{n}\right)$ is the so-called Cayley transform

$$
\begin{aligned}
\operatorname{Cay}\left(\tau \mathbf{A}_{n}^{\prime}\right): & =\left(\mathbf{I}_{6}-\tau \mathbf{A}_{n}^{\prime}\right)^{-1}\left(\mathbf{I}_{6}+\tau \mathbf{A}_{n}^{\prime}\right) \\
& =\left[\mathbf{I}_{6}+2 \tau\left(\mathbf{I}_{6}-\tau \mathbf{A}_{n}^{\prime}\right)^{-1} \mathbf{A}_{n}^{\prime}\right],
\end{aligned}
$$

where $\tau:=\Delta t / 2$, and $\mathbf{A}^{\prime}$ satisfying

$$
\left(\mathbf{A}^{\prime}\right)^{t} \mathbf{g}+\mathbf{g A}^{\prime}=0
$$

is a Lie algebra of the proper orthochronous Lorentz group $S O_{o}(5,1)$.

It is easy to check that the above transform $\operatorname{Cay}\left(\tau \mathbf{A}_{n}^{\prime}\right)$ preserves the properties (39)-(41) of the proper orthochronous Lorentz group, i.e., $\operatorname{Cay}\left(\tau \mathbf{A}_{n}^{\prime}\right) \in S_{o}(5$, 1). Therefore, the following transition formula

$$
\mathbf{X}_{n+1}=\exp [-\Delta t / \eta] \operatorname{Cay}\left(\tau \mathbf{A}_{n}^{\prime}\right) \mathbf{X}_{n}
$$

may be called a group preserving scheme. Obviously, it implies that

$$
\mathbf{X}_{n+1}^{t} \mathbf{g X}_{n+1}=\exp [-2 \Delta t / \eta] \mathbf{X}_{n}^{t} \mathbf{g} \mathbf{X}_{n}
$$

By Eq. (73) it follows that

$$
\mathbf{X}_{n}^{t} \mathbf{g} \mathbf{X}_{n}=0 \Rightarrow \mathbf{X}_{n+1}^{t} \mathbf{g} \mathbf{X}_{n+1}=0
$$

in the visco-elastoplastic phase, which says nothing but for every time increment the point $\mathbf{s}_{n+1}$ is located on the yield hypersphere, i.e., $\left\|\mathbf{s}_{n+1}\right\|=\sqrt{2} \tau_{y}$, if $\left\|\mathbf{s}_{n}\right\|=\sqrt{2} \tau_{y}$. In other words, the consistency condition is fulfilled exactly for every time stop in the visco-elastoplastic phase. This is what the conventional schemes of computational plasticity desired and failed to achieve directly in the stress space. Therefore, the new numerical scheme may be specifically called a consistency scheme.

Through some calculations the inverse of $\mathbf{I}_{6}-\tau \mathbf{A}^{\prime}$ is found to be

$$
\left(\mathbf{I}_{6}-\tau \mathbf{A}^{\prime}\right)^{-1}=\left[\begin{array}{cc}
\mathbf{B}+c \tau^{2} \mathbf{B} \mathbf{A}_{0}^{s} \mathbf{A}^{0} B & c \tau \mathbf{B} \mathbf{A}_{0}^{s} \\
c \tau \mathbf{A}^{0}{ }_{s} \mathbf{B} & c
\end{array}\right],
$$

where

$$
\mathbf{B}:=\left(\mathbf{I}_{5}-\tau \mathbf{A}^{\prime s}{ }_{s}\right)^{-1}, c:=\frac{1}{1-\tau^{2} \mathbf{A}^{0}{ }_{s} \mathbf{B} \mathbf{A}^{s}} .
$$

Substituting Eqs. (101), (84) and (75) $)_{1}$ into Eq. (98) we obtain the discretized transition matrix in the viscoelastoplastic phase,

$$
\operatorname{Cay}\left(\tau \mathbf{A}^{\prime}\right)=\left[\begin{array}{cc}
\mathbf{I}_{5}+2 c \tau^{3} \mathbf{B} \mathbf{A}_{0}^{s} \mathbf{A}_{s}^{0} \mathbf{B A}^{s}{ }_{s} & 2 c \tau^{3} \mathbf{B} \mathbf{A}_{0}^{s} \mathbf{A}_{s}^{0} \mathbf{B A}^{s}{ }_{0} \\
+2 \tau \mathbf{B} \mathbf{A}^{s}{ }_{s}+2 c \tau^{2} \mathbf{B} \mathbf{A}^{s}{ }_{0} \mathbf{A}^{0}{ }_{s} & +2 \tau \mathbf{B} \mathbf{A}^{s}{ }_{0} \\
2 c \tau^{2} \mathbf{A}^{0}{ }_{s} \mathbf{B A}^{s}{ }_{s}+2 c \tau \mathbf{A}^{0}{ }_{s} & 1+2 c \tau^{2} \mathbf{A}^{0}{ }_{s} \mathbf{B} \mathbf{A}^{s}
\end{array}\right] .
$$

Substituting the above $\operatorname{Cay}\left(\tau \mathbf{A}^{\prime}\right)$ with $t=t_{n}$ into Eq. (98) we obtain the mapping formula in the visco-elastoplastic phase.

The mapping in the viscoelastic phase is obtained by Eqs. (98) and (75) $)_{2}$ as in the following:

$\mathbf{X}_{n+1}=\left[\begin{array}{cc}\left.\exp \left[\frac{-\Delta t}{\eta}\right] \mathbf{I}_{5}+2 \tau \mathbf{B A}^{\prime s}{ }_{s}\right] & 2 \tau \exp \left[\frac{-\Delta t}{\eta}\right] \mathbf{B A}^{s}{ }_{0} \\ \mathbf{0}_{1 \times 5} & 1\end{array}\right] \mathbf{X}_{n}$,

from which it is obvious that $X_{n+1}^{0}=X_{n}^{0}$ in the viscoelastic phase. Once $\mathbf{X}_{n}$ is calculated at each time stop formula (90) gives the value of the response $\mathbf{s}_{n}$ at each time stop.

\section{Responses}

In order to gain the insight of the visco-elastoplastic behavior of material we apply the above numerical scheme to calculate the responses under certain inputs and initial conditions. Let us first consider the simplest case where both $\mathbf{D}$ and $\mathbf{W}$ are constant matrices. The material constants used in the calculations were $G=50$, $000 \mathrm{MPa}$ and $\tau_{y}=500 \mathrm{MPa}$. We applied three cycles, each cycle consisting of constant $\mathbf{D}$ and $\mathbf{W}$ for one second and then negative constant $\mathbf{D}$ and $\mathbf{W}$ for another one second, with

$\mathbf{D}=\left[\begin{array}{ccc}0.002 & 0.009 & 0.005 \\ 0.009 & -0.001 & 0.004 \\ 0.005 & 0.004 & -0.001\end{array}\right], \mathbf{W}=\left[\begin{array}{ccc}0 & 0.001 & 0.002 \\ -0.001 & 0 & -0.005 \\ -0.002 & 0.005 & 0\end{array}\right]$.

Figure 1 shows the responses for different $\eta$ 's with $\eta=0.5,1,5$, where the initial stresses were taken to be $s^{11}=50 \mathrm{MPa}, s^{22}=20 \mathrm{MPa}, s^{23}=-100 \mathrm{MPa}, s^{13}=-40$ $\mathrm{MPa}$, and $s^{12}=30 \mathrm{MPa}$. For the case $\eta=0.5$, the input $\mathbf{D}$ is not large enough to render $\mathbf{S} \cdot \mathbf{D}>\gamma_{y} \tau_{y} / \eta$, such that the responses are of viscoelasticity. It can be seen that the hysteretic loops for viscoelasticity have sharp tips at two endpoints of the stress-strain curve, and that after the first cycle the hysteresis loops are stabilized and have almost the identical shape. For the other two cases $\eta=1$ and $\eta=5$ there occur several times the phase transitions between viscoelasticity and visco- 

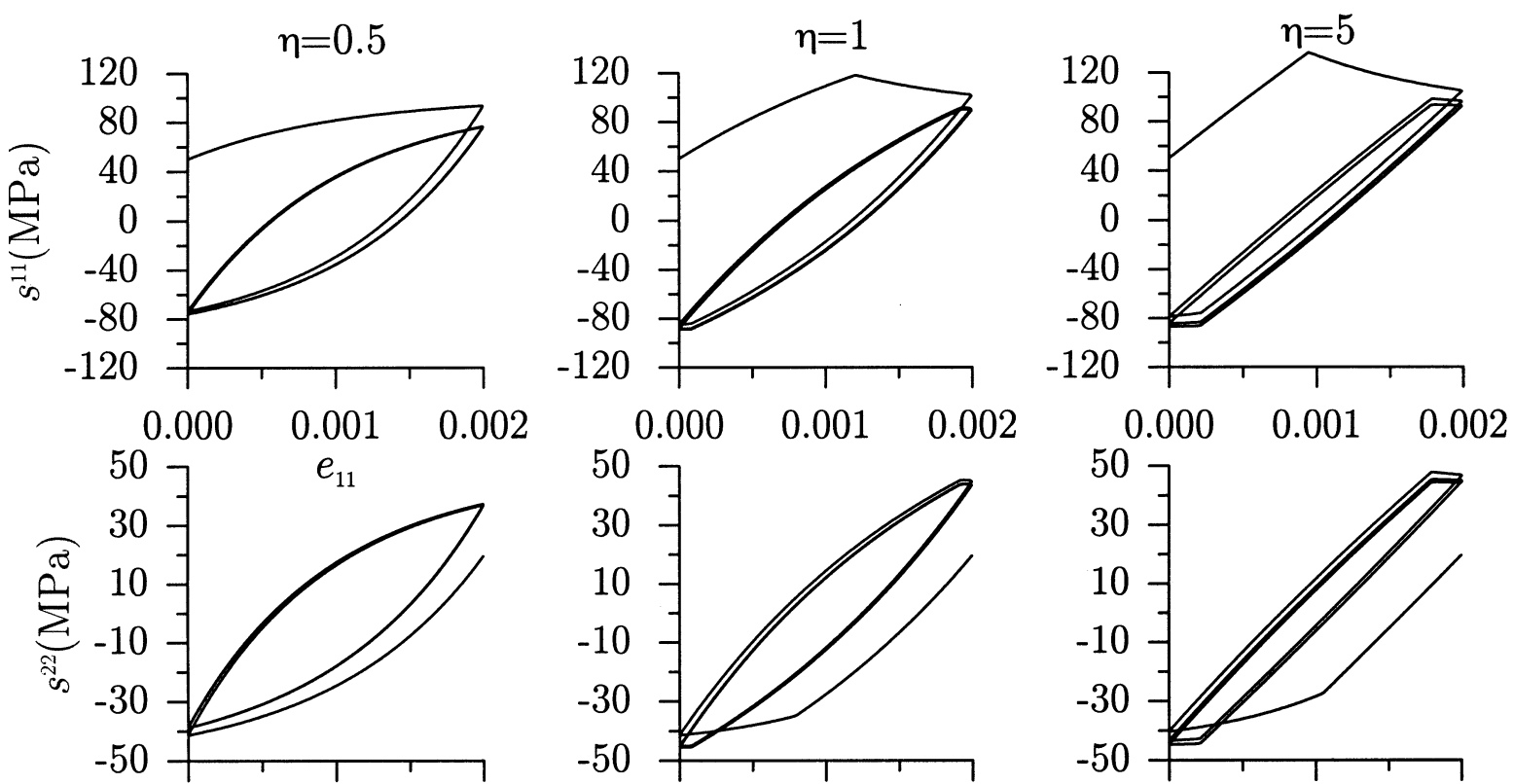

$\begin{array}{lll}-0.0010 & -0.0005 & 0.0000\end{array}$

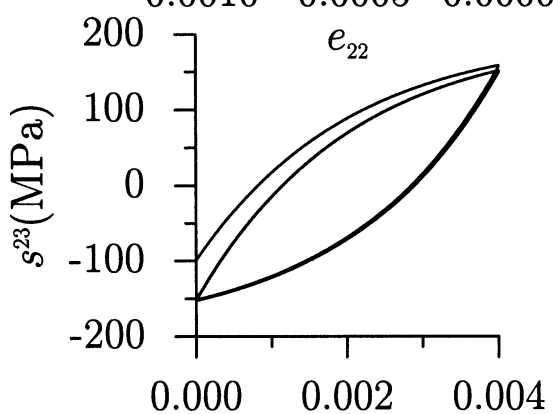

$\begin{array}{lll}-0.0010 & -0.0005 & 0.0000\end{array}$
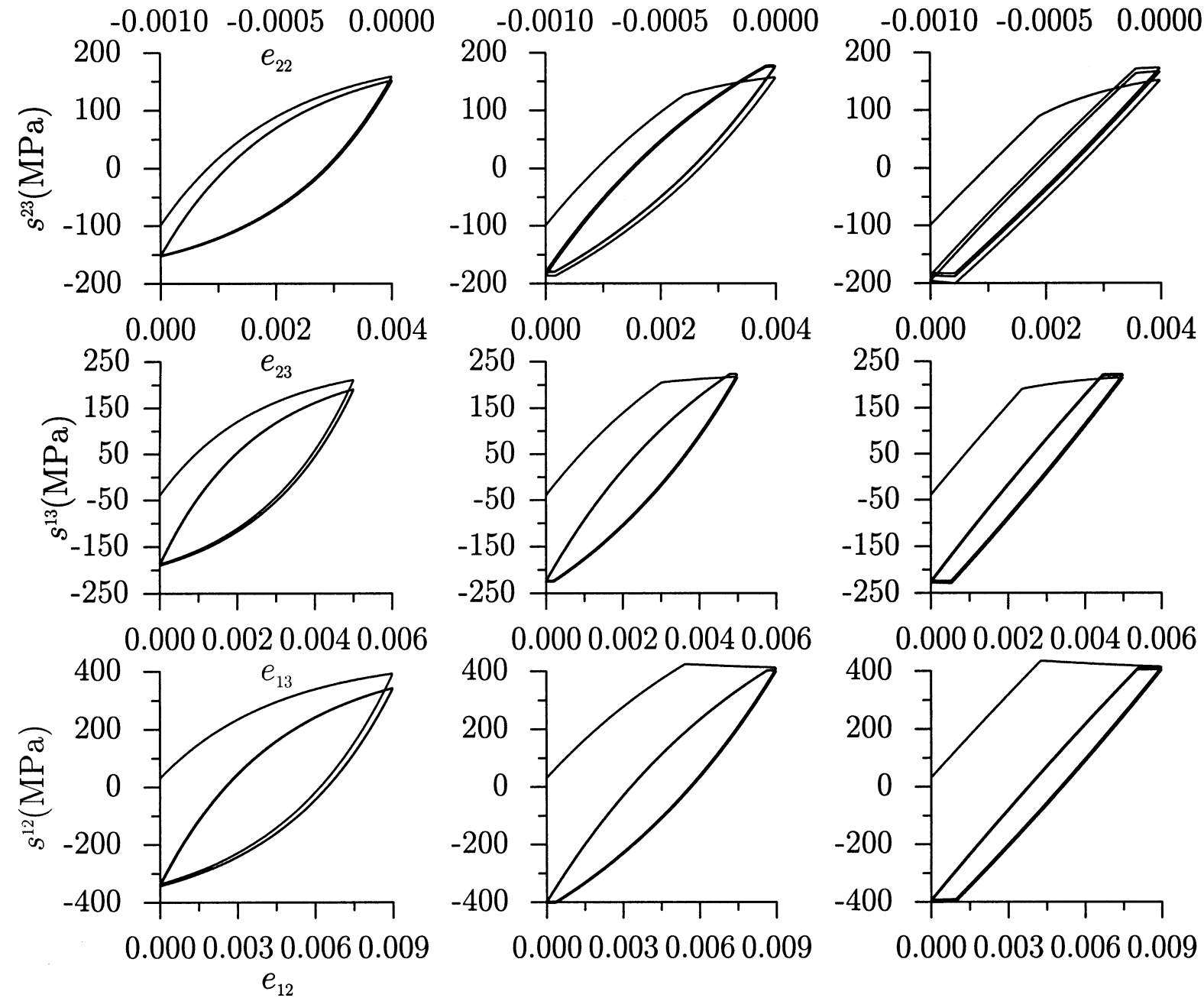

Fig. 1. The cyclic response curves $\left(\boldsymbol{s}^{11}, e_{11}\right),\left(\boldsymbol{s}^{22}, e_{22}\right),\left(\boldsymbol{s}^{23}, e_{23}\right),\left(\boldsymbol{s}^{13}, e_{13}\right)$, and $\left(\boldsymbol{s}^{12}, e_{12}\right)$ under constant deformation rate and spin for different $\eta$ 's with $\eta=$ $0.5,1,5$. 

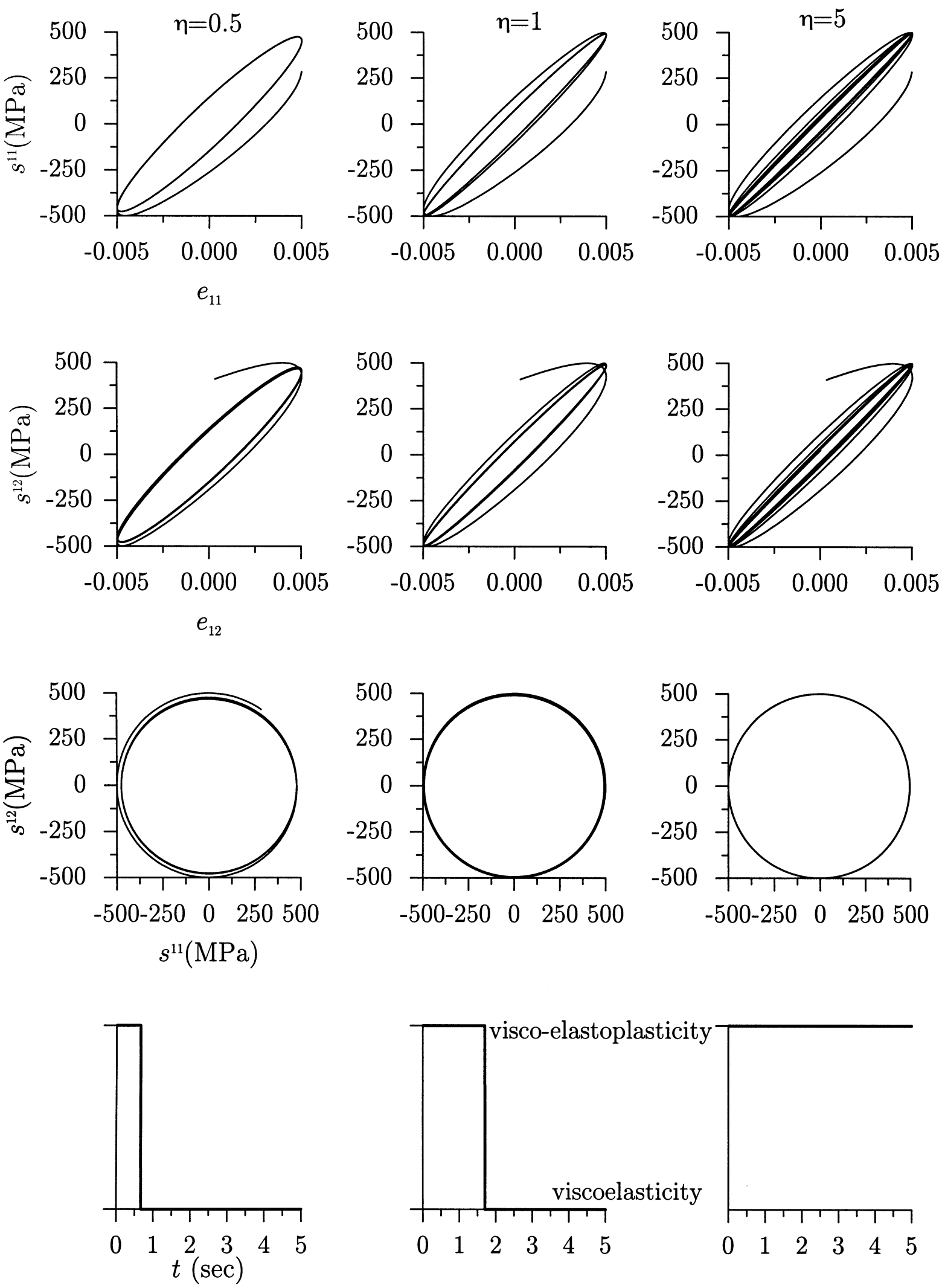

Fig. 2. The cyclic response curves of $s^{11}$ and $s^{12}$ under constant spin and circular strain path with $e_{11}=0.005 \cos \omega t$ and $e_{12}=0.005$ sin $\omega t$ for different $\eta$ 's with $\eta=0.5,1,5$. 
elastoplasticity. Therefore, in the stabilized hysteretic loops the original sharp tips for $\eta=0.5$ are now flattened due to the incipience of visco-elastoplasticity. Figure 2 shows the responses under the input $D_{11}=$ $-0.005 \omega \sin \omega t 1 / \mathrm{sec}, D_{12}=0.005 \omega \cos \omega t 1 / \mathrm{sec}$, where $\omega=2 \pi$, and the other components of $\mathbf{D}$ are zeros. $\mathbf{W}$ is the same as above. The initial stresses were taken to be located on the yield surface with $s^{11}=300 \mathrm{MPa}, s^{22}=0$ $\mathrm{MPa}, s^{23}=0 \mathrm{MPa}, s^{13}=0 \mathrm{MPa}$, and $s^{12}=400 \mathrm{MPa}$. However, for the cases $\eta=0.5$ and 1 the responses are of viscoelasticity after the time $0.668 \mathrm{sec}$ and $1.704 \mathrm{sec}$ respectively, as shown in the figures of the last row. The stress path $\left(s^{11}, s^{12}\right)$ plotted in the figures at the third row shows that in the viscoelastic phase the case $\eta=$ 0.5 has smaller oscillating amplitude than $\tau_{y}$, but the case $\eta=1$ nearly has the same oscillating amplitude $\tau_{y}$ as the case $\eta=5$ has. The resulting hysteresis loops are ellipses in the planes $\left(s^{11}, e_{11}\right)$ and $\left(s^{12}, e_{12}\right)$. It reveals that the larger the relaxation time $\eta$ is, the thinner the hysteresis loop will be. For the cases $\eta=5$ the responses are of visco-elastoplasticity after the initial time. It shows that the consistency scheme supplied a completely faithful result of the consistency condition $\left(s^{11}\right)^{2}+\left(s^{12}\right)^{2}=\tau_{y}^{2}$. It can be seen that the hysteresis loops for viscoelasticity are stabilized more faster than the ones for the visco-elastoplasticity. In the above figures each component of the strain is calculated by the integration of each component of $\mathbf{D}$.

\section{CONCLUDING REMARKS}

We derived the algebraic foundation of dynamical system (35). It is the composition of the $g$-based Jordan and Lie algebras. The group symmetry of this sort dynamical system was proved to be the projection of the dilation proper orthochronous Lorentz transformation $\mathrm{PDSO}_{0}(n, 1)$.

Specialized to dimension $n=5$, we investigated internal symmetry inherent in the constitutive model of visco-elastoplasticity with large deformation. Even though the constitutive equations as shown in Eq. (59) are nonlinear in the deviatoric stress space of $\mathbf{s}$, they can be converted to a linear system $\dot{\mathbf{X}}=\mathbf{A X}$ in the $(5+1)$ dimensional augmented stress space of $\mathbf{X}$. In the augmented stress space not only the nonlinearity of the model is unfolded, but also an internal spacetime structure of the Minkowskian type is brought out. The control tensor $\mathbf{A}$ for the visco-elastoplastic phase was proved to be an element of the real Lie algebra of the dilation proper orthochronous Lorentz group $\mathrm{DSO}_{o}(5$, 1), and the fundamental solution of the system $\dot{\mathbf{X}}=\mathbf{A X}$ with the visco-elastoplastic phase $\mathbf{A}$ was shown to be an element of the dilation proper orthochronous Lorentz group. To account for both the visco-elastoplastic and viscoelastic phases we constructed a composite space endowed with a Minkowskian metric on the cone but with a Euclidean metric on each of the discs inside the cone. As a result we found that the visco-elastoplastic model with large deformation possesses two kinds of symmetry: $D S E(5)$ in the viscoelastic phase and $P D S O_{o}$ $(5,1)$ in the visco-elastoplastic phase, and has symmetry switching between the two depending on the control input and the relaxation time.

Based on the symmetry study, a numerical scheme which preserves the group properties for every time increment was developed. This group preserving scheme may be specifically called a consistency scheme, since it is capable, among other benefits derivable from the group properties, of updating the stress point to be automatically located on the yield surface at the end of each time increment in the visco-elastoplastic phase without any iterative calculations, that is, the consistency condition is fulfilled automatically and exactly. Since the consistency scheme is easy to implement numerically and has high computational efficiency and accuracy, it is highly recommended for engineering applications.

\section{REFERENCES}

1. Liu, C.-S., "A Jordan algebra and dynamic system with associator as vector field," Int. J. Non-Linear Mech., Vol. 35, pp. 421-429 (2000).

2. Jacobson, N., Basic Algebra I, 2nd ed., Freeman, San Francisco (1981).

3. Schafer, R. D., An Introduction to Nonassociative Algebra, Dover, New York (1995).

4. Hong, H.-K. and Liu, C.-S., "Internal symmetry in the constitutive model of perfect elastoplasticity," Int. J. Non-Linear Mech., Vol. 35, pp. 447-466 (2000).

5. Hong, H.-K. and Liu, C.-S., "Prandtl-Reuss elastoplasticity: on-off switch and superposition formulae," Int. J. Solids Struct., Vol. 34, pp. 4281-4304 (1997).

6. Landau, L. D. and Lifshitz, E. M., "On the theory of the dispersion of magnetic permeability in ferromagnetic bodies," Phys. Z. Sowjetunion, Vol. 8, pp. 153-169 (1935).

7. Liu, C.-S., "Orientation control of particles and fibers for polymeric liquids and fiber reinforced materials," $J$. Chinese Inst. Engineers, Vol. 20, pp. 443-456 (1997).

8. Hong, H.-K. and Liu, C.-S., "Internal symmetry in bilinear elastoplasticity," Int. J. Non-Linear Mech., Vol. 34, pp. 279-288 (1999).

9. Hong, H.-K. and Liu, C.-S., "Lorentz group $\mathrm{SO}_{o}(5,1)$ for perfect elastoplasticity with large deformation and a consistency numerical scheme," Int. J. Non-Linear Mech., Vol. 34, pp. 1113-1130 (1999). 
10. Liu, C.-S. and Hong, H.-K., "Using comparison theorem to compare corotational stress rates in the model of perfect elastoplasticity," Int. J. Solids Struct., Vol. 38, pp. 2969-2987 (2001).

11. Yaglom, I. M., Complex Numbers in Geometry, Academic, New York (1968).

12. Zeeman, E. C., "Causality implies the Lorentz group," J. Math. Phys., Vol. 5, pp. 490-493 (1964).

13. Prandtl, L., "Spannungsverteilung in plastischen koerpern," in Proceedings of the 1st International Congress on Applied Mechanics, Delft (1924) 43-54.

14. Reuss, E., "Beruecksichtigung der elastischen formaenderungen in der plastizitaetstheorie," Zeits. angew. Math. Mech. (ZAMM), Vol. 10, pp. 266-274 (1930).

15. Christension, R. M., Theory of Viscoelasticity, 2nd ed., Academic, New York (1982).

\section{$g$ 數約旦代數與李代數在秥彈塑性組 成律的應用}

$$
\text { 劉進賢 }
$$

國立臺灣海洋大學機械與輪機工程學系

$$
\text { 摘 要 }
$$

透過矩陣表示, 本文證明文獻 $[1]$ 的 $g$ 數約旦代 數, 在交換子乘積運算下構成g數李代數。據此我們 提出一個組合 $g$ 數約旦代數與李代數的微分動態系 統, 並證明該系統具有内部對稱群 $D S O_{o}(n, 1)$, 其射 影群記爲 $P D S O_{o}(5,1)$ 。運用這個觀念我們推導大變 形黍彈塑性組成律的線性表示。根據不可約表示證明 秥彈塑相時其向量空間作用群爲射影收縮正常保時勞 倫茲群, 記爲 $P D S O_{o}(5,1)$; 反之, 黏彈相時其作用 群爲收縮特殊歐氏群, 記爲 $D S E(5)$ 。兩種對稱群之 間的切換和輸入路徑及鑾弛時間有關。根據對稱群性 質本文發展出在每一時間步都保證霂足一致性條件的 數值計算方法, 此法保持本模式在敉彈塑相時的内部 對稱性 $P D S O_{o}(5,1)$, 因此使得應力點在每一時間步 都自動落在降伏面上, 不需要額外的迭代計算。 